\title{
Canonical quantization of a two-dimensional model with anomalous breaking of gauge invariance
}

\author{
H. O. Girotti, ${ }^{*}$ H. J. Rothe, and K. D. Rothe \\ Institut für Theoretische Physik der Universität Heidelberg, Philosophenweg 16, D-6900 Heidelberg, West Germany
}

(Received 13 August 1985)

\begin{abstract}
We investigate in detail the operator quantum dynamics of a two-dimensional model exhibiting anomalous breaking of gauge invariance. The equal-time algebra is systematically obtained by using the Dirac-bracket formalism for constrained systems. For certain values of the regularization parameter the system is shown to undergo drastic changes. For the value of the parameter corresponding to the chiral Schwinger model no operator solutions are found to exist.
\end{abstract}

\section{INTRODUCTION}

In a recent paper $^{1}$ the interesting possibility has been raised that in gauge theories involving chiral fermions the local gauge symmetry present on the classical level may not survive quantization and may provide an alternative mechanism of mass generation of the vector bosons in weak interactions. Even if local gauge invariance is broken on the quantum level, the current coupling to the gauge field may still be conserved, in which case no inconsistency with the equation of motion

$$
\partial_{\mu} F^{\mu v}=J^{v}
$$

arises. An example of this is provided by the $(1+1)$ dimensional chiral model defined by the Lagrangian ${ }^{1,2}$

$$
\mathscr{L}=-\frac{1}{4} F^{\mu \nu} F_{\mu \nu}+\bar{\psi}\left[i \partial+e \sqrt{\pi} \Lambda\left(1+i \gamma_{5}\right)\right] \psi .
$$

At the classical level $\mathscr{L}$ is invariant under the local gauge transformation

$$
\begin{aligned}
& A_{\mu}(x) \rightarrow A_{\mu}(x)+\partial_{\mu} \alpha(x), \\
& \psi(x) \rightarrow e^{2 i e \sqrt{\pi} \alpha(x) P_{+}} \psi(x), \\
& \bar{\psi}(x) \rightarrow \bar{\psi}(x) e^{-2 i e \sqrt{\pi} \alpha(x) P_{-}},
\end{aligned}
$$

where

$$
P_{ \pm}=\frac{1}{2}\left(1 \pm i \gamma_{5}\right) \text {. }
$$

On the quantum level, it was shown in Ref. 1 that the dynamics of the model is described by the local effective Lagrangian

$$
\begin{aligned}
\mathscr{L}_{\text {eff }}= & -\frac{1}{4} F^{\mu v} F_{\mu \nu}+\frac{1}{2}\left(\partial^{\mu} \phi\right)\left(\partial_{\mu} \phi\right) \\
& +e\left(g^{\mu \nu}-\epsilon^{\mu v}\right) \partial_{\mu} \phi A_{v}+\frac{1}{2} a e^{2} A_{\mu} A^{\mu},
\end{aligned}
$$

where $a$ is a constant related to the different regularization procedures used for calculating the fermionic determinant. As was pointed out in the above-mentioned reference, no choice of $a$ can restore the original gauge symmetry.

The dynamics arising from (1.3) was partially studied in Ref. 1 for the case $a>1$. In particular, the solutions appear to be singular for $a=1$. A similar statement applies to the case $a=0$. It is thus unclear whether the theory exists for these values of $a$.

One can convince oneself that the Lagrangian (1.3) describes a constrained system. Hence, a systematic opera- tor quantization of the theory can best be carried out using a Dirac-bracket formalism. ${ }^{3}$ As we shall show the constraints are second class for all values of $a$, which clearly indicates that the original symmetry (1.2) does not survive quantization.

In this paper we have canonically implemented the quantum dynamics of the model for $a \geq 0$. The equaltime commutator algebra studied in Sec. II turns out to be singular for $a=1$, while the operator solutions become singular for $a=1$ and 0 . Hence, these two cases will be treated separately in the following sections. For the case $a>1$ it is rigorously shown that the equal-time commutator algebra, abstracted from the Dirac brackets for the original fields $\phi$ and $A^{\mu}$, can be realized in terms of two free scalar fields. The above-mentioned singularity at $a=1$ now manifests itself in the equal-time commutators.

Although the limit $a \rightarrow 1$ does not exist, a consistent quantum theory is obtained by setting $a=1$ in the Lagrangian given by Eq. (1.3). This case is studied in Sec. III. One of the peculiar features of the system is that, for this value of $a$, the number of constraints doubles and hence one is led to a completely new equal-time commutator algebra. The theory remains a second-class theory and therefore the local gauge symmetry remains broken.

For $a<1$ the model develops tachyons. On the other hand, the equal-time commutator algebra possesses a well-defined limit $a \rightarrow 0$. Nevertheless there exist no operator solutions compatible with the equal-time commutator algebra for $a=0$, as we shall demonstrate in Sec. IV. Some final remarks and the conclusions are contained in Sec. $V$.

\section{CANONICAL QUANTIZATION FOR $a>1$}

From the Lagrangian (1.3) we obtain for the momenta canonically conjugate to the coordinates $A^{0}, A^{1}$, and $\phi$

$$
\begin{aligned}
& \Omega_{1} \equiv \pi_{0}=0, \\
& \pi_{1}=F^{01}=\partial^{0} A^{1}-\partial^{1} A^{0}, \\
& \pi_{\phi}=\partial^{0} \phi+e\left(g_{0 \mu}-\epsilon_{0 \mu}\right) A^{\mu},
\end{aligned}
$$

where $\Omega_{1}$ is the primary constraint. The total Hamiltonian $H_{T}$ following from (1.3) in the standard way is ${ }^{4}$

$$
H_{T}=H+\int d y^{1} \xi^{0}\left(y^{1}\right) \pi_{0}\left(y^{1}\right),
$$

where 


$$
\begin{aligned}
H=\int d y^{1} & \frac{1}{2} \pi_{1}^{2}-A^{0} \partial^{1} \pi_{1}+\frac{1}{2} \pi_{\phi} \pi_{\phi}+\frac{1}{2}\left(\partial^{1} \phi\right)\left(\partial^{1} \phi\right)-\frac{a e^{2}}{2} A_{\mu} A^{\mu}+\frac{1}{2} e^{2}\left(g_{0 \mu}-\epsilon_{0 \mu}\right)\left(g_{0 v}-\epsilon_{0 v}\right) A^{\mu} A^{v} \\
& \left.-e \pi_{\phi}\left(g_{0 \nu}-\epsilon_{0 v}\right) A^{v}-e\left(g_{1 v}-\epsilon_{1 v}\right)\left(\partial^{1} \phi\right) A^{v}\right)
\end{aligned}
$$

and $\xi^{0}\left(y^{1}\right)$ is a (so far arbitrary) Lagrange multiplier.

Following the algorithm of Dirac, ${ }^{3}$ we find that the requirement of persistence in time of the primary constraint leads to the existence of the secondary constraint

$$
\Omega_{2} \equiv \partial^{1} \pi_{1}+a e^{2} A^{0}-e^{2}\left(g_{0 v}-\epsilon_{0 v}\right) A^{v}+e \pi_{\phi}-e \partial^{1} \phi \approx 0 .
$$

In the case $a>1$ no new constraints are generated by requiring persistence in time of this secondary constraint. Indeed, since the Poisson brackets

$$
\left\{\Omega_{1}\left(x^{1}\right), \Omega_{2}\left(y^{1}\right)\right\}=-e^{2}(a-1) \delta\left(x^{1}-y^{1}\right)
$$

does not vanish for $a>1$, the constraints are second class, and hence the persistence in time of $\Omega_{2} \approx 0$ merely serves to determine the Lagrange multiplier $\xi^{0}$. The nonvanishing of (2.6) implies that the local gauge invariance (1.2) has been broken at the level of the effective Lagrangian (1.3). One might erroneously conclude that for analogous reasons the local gauge symmetry remains unbroken for $a=1$. However, this is not so, as will be shown in the following section.

The canonical quantization of the theory is achieved by abstracting the equal-time commutators from the corresponding Dirac brackets. The nonvanishing commutators read

$$
\begin{aligned}
& {\left[A^{1}\left(x^{1}\right), \pi_{1}\left(y^{1}\right)\right]=i \delta\left(x^{1}-y^{1}\right),} \\
& {\left[\phi\left(x^{1}\right), \pi_{\phi}\left(y^{1}\right)\right]=i \delta\left(x^{1}-y^{1}\right),} \\
& {\left[A^{0}\left(x^{1}\right), A^{1}\left(y^{1}\right)\right]=\frac{i}{e^{2}(a-1)} \partial_{x}^{1} \delta\left(x^{1}-y^{1}\right),} \\
& {\left[A^{0}\left(x^{1}\right), \phi\left(y^{1}\right)\right]=\frac{i}{e(a-1)} \delta\left(x^{1}-y^{1}\right),} \\
& {\left[A^{0}\left(x^{1}\right), \pi_{1}\left(y^{1}\right)\right]=\frac{i}{a-1} \delta\left(x^{1}-y^{1}\right),} \\
& {\left[A^{0}\left(x^{1}\right), \pi_{\phi}\left(y^{1}\right)\right]=\frac{i}{e(a-1)} \partial_{x}^{1} \delta\left(x^{1}-y^{1}\right) .}
\end{aligned}
$$

We remark that, in particular, all equal-time commutators involving a constraint vanish.

From (2.7) it follows that operator-ordering problems exist in the Hamiltonian (2.4b). We ensure the Hermiticity of $H$ by replacing ordinary operator products by symmetrized products. One can then easily show that the corresponding Heisenberg equations of motion together with the constraints are completely equivalent to the Lagrange equations of motion derived from (1.3):

$$
\begin{aligned}
& \square \phi=-e\left(g^{\mu v}-\epsilon^{\mu v}\right) \partial_{\mu} A_{v}, \\
& \square A^{\mu}-\partial^{\mu} \partial_{v} A^{v}+a e^{2} A^{\mu}=-e\left(g^{\mu v}+\epsilon^{\mu v}\right) \partial_{\nu} \phi .
\end{aligned}
$$

The most general solution to these equations can be shown to be $\mathrm{b}^{1}$

$$
A^{\mu}=-\frac{1}{a e}\left[\partial^{\mu} \phi+(1-a) \epsilon^{\mu v} \partial_{\nu} \phi-a \epsilon^{\mu \nu} \partial_{v} h\right],
$$

where

$$
\phi=\sigma-h,
$$

with

$$
\begin{aligned}
& \left(\square+m^{2}\right) \sigma=0, \\
& \square h=0,
\end{aligned}
$$

and

$$
m^{2}=\frac{a^{2} e^{2}}{a-1} .
$$

Although $\sigma$ and $h$ are free fields they may not be independent. Their precise interrelationship is determined by the initial conditions, i.e., the Lagrangian version of the canonical equal-time commutators (2.7) (including the vanishing ones). The commutators we shall need are

$$
\begin{aligned}
& {\left[A^{0}\left(x^{1}\right), A^{1}\left(y^{1}\right)\right]=\frac{i}{e^{2}(a-1)} \partial_{x}^{1} \delta\left(x^{1}-y^{1}\right),} \\
& {\left[A^{0}\left(x^{1}\right), \phi\left(y^{1}\right)\right]=\frac{i}{e(a-1)} \delta\left(x^{1}-y^{1}\right),} \\
& {\left[A^{0}\left(x^{1}\right), \partial^{0} A^{1}\left(y^{1}\right)\right]=\frac{i}{a-1} \delta\left(x^{1}-y^{1}\right),} \\
& {\left[A^{1}\left(x^{1}\right), \partial^{0} A^{1}\left(y^{1}\right)\right]=i\left(1-\frac{1}{e^{2}(a-1)} \partial_{x}^{1} \partial_{x}^{1}\right) \delta\left(x^{1}-y^{1}\right),} \\
& {\left[A^{0}\left(x^{1}\right), \partial^{0} \phi\left(y^{1}\right)\right]=0,} \\
& {\left[A^{1}\left(x^{1}\right), \partial^{0} \phi\left(y^{1}\right)\right]=-\frac{i}{e(a-1)} \partial_{x}^{1} \delta\left(x^{1}-y^{1}\right),} \\
& {\left[\phi\left(x^{1}\right), \partial^{0} A^{1}\left(y^{1}\right)\right]=\frac{i}{e(a-1)} \partial_{x}^{1} \delta\left(x^{1}-y^{1}\right),} \\
& {\left[\phi\left(x^{1}\right), \partial^{0} \phi\left(y^{1}\right)\right]=i \frac{a}{a-1} \delta\left(x^{1}-y^{1}\right),} \\
& {\left[\partial^{0} \phi\left(x^{1}\right), \partial^{0} \phi\left(y^{1}\right)\right]=0 .}
\end{aligned}
$$

Using the solution (2.9) and (2.10), it follows from the above commutation relations that $\sigma$ and $h$ are in fact independent fields with $h$ satisfying standard canonical commutation relations

$$
\begin{aligned}
& {\left[h\left(x^{1}\right), h\left(y^{1}\right)\right]=0,} \\
& {\left[h\left(x^{1}\right), \partial^{0} h\left(y^{1}\right)\right]=i \delta\left(x^{1}-y^{1}\right),}
\end{aligned}
$$

and $\sigma$ satisfying 


$$
\begin{aligned}
& {\left[\sigma\left(x^{1}\right), \sigma\left(y^{1}\right)\right]=0,} \\
& {\left[\sigma\left(x^{1}\right), \partial^{0} \sigma\left(y^{1}\right)\right]=\frac{i}{a-1} \delta\left(x^{1}-y^{1}\right) .}
\end{aligned}
$$

From here and (2.10) it follows that $\phi$ and $h$ are not independent fields.

This algebra provides a Fock-space realization for the solution (2.9) and (2.10), and it is a simple exercise to show that

$$
\left\langle 0\left|T\left[A^{\mu}(x) A^{v}(y)\right]\right| 0\right\rangle=i\left[-g^{\mu \nu}+\frac{\partial^{\mu} \partial^{v}}{a-1}\left(\frac{1}{e^{2}}-\frac{2}{\square}\right)-\frac{1}{a-1}\left[\frac{\partial^{\mu} \tilde{\partial}^{\nu}+\partial^{\nu} \tilde{\partial}^{\mu}}{\square}\right]\right] D_{F}(x-y)+\text { contact terms }
$$

which, aside from the contact terms, agrees with the result of Ref. 1. In (2.17) we have used the notation $\widetilde{\partial}^{\mu}$ $=e^{\mu v} \partial_{v} ; D_{F}(x-y)$ is just the usual two-dimensional propagator of a free massless scalar field.

We note that the commutation relations (2.7) and (2.14) as well as the propagator (2.17) are singular at $e=0$, so that the theory does not admit a perturbative expansion in the coupling constant. In particular, the theory does not approach a free field theory in the limit $e \rightarrow 0$.

Finally we observe that for $0<a<1$ the theory involves tachyons. ${ }^{1}$ Hence, we shall limit ourselves in the following sections to a discussion of the singular cases $a=1$ and 0 .

\section{CANONICAL QUANTIZATION FOR $a=1$}

As seen from Eq. (2.6), the Poisson brackets $\left\{\Omega_{1}\left(x^{1}\right), \Omega_{2}\left(x^{2}\right)\right\}=0$ for $a=1$. Hence, only two possibilities exist: (a) the theory becomes a gauge theory, or (b) the theory remains second class; then, additional constraints must emerge. The first possibility is ruled out by a simple inspection of the Lagrangian (1.3). Thus, we expect to find new constraints.

Indeed, by requiring persistence in time of

$$
\Omega_{2}=\partial^{1} \pi_{1}-e\left(e A^{1}+\pi_{\phi}-\partial^{1} \phi\right) \approx 0,
$$

one obtains the new secondary constraint

$$
\Omega_{3} \equiv \pi_{1} \approx 0,
$$

which expresses the vanishing of the field tensor. In turn, the condition $\partial^{0} \Omega_{3} \approx 0$ leads to

$$
\Omega_{4} \equiv e\left(A^{0}+A^{1}\right)+\left(e A^{1}-\pi_{\phi}+\partial^{1} \phi\right) \approx 0 .
$$

Since

$$
\left\{\Omega_{1}\left(x^{1}\right), \Omega_{4}\left(y^{1}\right)\right\} \neq 0
$$

the requirement $\partial^{0} \Omega_{4} \approx 0$ just determines the Lagrange multiplier in (2.4a). Therefore, there are no further constraints, and one easily verifies that all constraints are second class. This drastic change in the constraint structure evidently is linked to the singular behavior of the equal-time algebra (2.7) in the limit $a=1$.

Proceeding as in Sec. II, we obtain this time for the nonvanishing equal-time commutators the following expressions:

$$
\begin{aligned}
& {\left[A^{0}\left(x^{1}\right), A^{1}\left(y^{1}\right)\right]=\frac{2 i}{e^{2}} \partial_{x}^{1} \delta\left(x^{1}-y^{1}\right),} \\
& {\left[A^{0}\left(x^{1}\right), \phi\left(y^{1}\right)\right]=\frac{i}{e} \delta\left(x^{1}-y^{1}\right),} \\
& {\left[A^{0}\left(x^{1}\right), \pi_{\phi}\left(y^{1}\right)\right]=\frac{i}{e} \partial_{x}^{1} \delta\left(x^{1}-y^{1}\right),} \\
& {\left[A^{1}\left(x^{1}\right), \phi\left(y^{1}\right)\right]=-\frac{i}{e} \delta\left(x^{1}-y^{1}\right),} \\
& {\left[A^{1}\left(x^{1}\right), \pi_{\phi}\left(y^{1}\right)\right]=-\frac{i}{e} \partial_{x}^{1} \delta\left(x^{1}-y^{1}\right),} \\
& {\left[\phi\left(x^{1}\right), \pi_{\phi}\left(y^{1}\right)\right]=i \delta\left(x^{1}-y^{1}\right) .}
\end{aligned}
$$

We remark that all equal-time commutators involving any of the constraints vanish. In particular $\left[A^{1}\left(x^{1}\right), \pi_{1}\left(y^{1}\right)\right]$ $=0$.

For $a=1$ the Hamiltonian (2.4b) can be rewritten in the following form:

$$
\begin{aligned}
H= & H\left[\phi, \pi_{\phi}\right] \\
& +\int d x^{1}\left[\frac{1}{2} \Omega_{3} \Omega_{3}+A^{1} \partial^{1} \Omega_{3}-\left(A^{0}+A^{1}\right) \Omega_{2}\right],
\end{aligned}
$$

where

$$
H\left[\phi, \pi_{\phi}\right]=\frac{1}{2} \int d x^{1}\left[\pi_{\phi} \pi_{\phi}+\left(\partial^{1} \phi\right)\left(\partial^{1} \phi\right)\right] .
$$

The second term in (3.5a) vanishes strongly and the spectrum of the Hamiltonian is that of a free massless field. From the constraints (3.1)-(3.3) one finds

$$
A^{0}=-A^{1}=-\frac{2}{e} \partial_{+} \phi
$$

where $\partial_{ \pm}$denotes the derivatives with respect to the light-cone variables $x^{ \pm}=x^{0} \pm x^{1}$. Since $\phi$ is a massless free field, Eq. (3.6) shows that $A^{\mu}$ is a regressive wave propagating with the velocity of light. The Lagrange equations of motion implied by (3.4) and (3.5a) are

$$
\begin{aligned}
& J^{v} \equiv \partial_{\mu} F^{\mu v}=-e^{2} A^{v}-e\left(g^{\nu \mu}+\epsilon^{\nu \mu}\right) \partial_{\mu} \phi, \\
& \square \phi=0 .
\end{aligned}
$$

From (3.6) and (3.7) one sees that

$$
J^{\mu}=0 \text {, }
$$

which is in agreement with (3.2). Hence, the quantum theory exists for $a=1$, and it cannot be obtained as the limit $a \rightarrow 1$ from the theory discussed in Sec. II. In particular the $A^{\mu}$ propagator turns out to be given by the fi- 
nite result

$$
\begin{aligned}
\left\langle 0\left|T\left[A^{\mu}(x) A^{v}(y)\right]\right| 0\right\rangle=- & \frac{i}{e^{2}}\left(2 \partial^{\mu} \partial^{v}+\partial^{\mu} \tilde{\partial}^{v}+\partial^{v} \tilde{\partial}^{\mu}\right) \\
& \times D_{F}(x-y),
\end{aligned}
$$

whereas the limit $a \rightarrow 1$ of the propagator (2.17) does not exist.

\section{THE CASE $a=0$}

From inspection of (2.6) it follows that the constraint structure for $a=0$ is the same as in the case $a>1$. Thus the equal-time commutators for $a=0$ are those of Eq. (2.7) after setting $a=0$. The Hamiltonian (2.4b) with $a=0$ leads in a way analogous to that of Sec. II to the Lagrange equations

$$
\begin{aligned}
& \square \phi=-e\left(g^{\mu v}-\epsilon^{\mu v}\right) \partial_{\mu} A_{v}, \\
& \square A^{\mu}-\partial^{\mu}\left(\partial_{v} A^{v}\right)=-e\left(g^{\mu v}+\epsilon^{\mu v}\right) \partial_{\nu} \phi .
\end{aligned}
$$

It is interesting to note that, even though on the level of the effective Lagrangian (1.3) with $a=0$ the original local gauge invariance (1.2) is completely broken, there exists a residual gauge invariance on the level of the equations of motion (4.1) corresponding to the transformations

$$
\begin{aligned}
& A^{\mu} \rightarrow A^{\mu}+\partial^{\mu} \Lambda, \quad \square \Lambda=0, \\
& A^{\mu} \rightarrow A^{\mu}+\epsilon^{\mu \nu} \partial_{v} \tilde{\Lambda}, \square \tilde{\Lambda}=0 .
\end{aligned}
$$

From (4.1a) and (4.1b) it immediately follows that

$$
\begin{aligned}
& \square \phi=0, \\
& \partial^{\mu} A_{\mu}=\epsilon^{\mu v} \partial_{\mu} A_{v} .
\end{aligned}
$$

To look for solutions of Eqs. (4.1) we make use of the fact that in $1+1$ dimensions $A^{\mu}$ can always be written in the form

$$
A^{\mu}=\epsilon^{\mu \nu} \partial_{\nu} \chi+\partial^{\mu} \eta
$$

which enables one to recast $(4.3 \mathrm{~b})$ and the curl of $(4.1 \mathrm{~b})$ in the form

$$
\begin{aligned}
& \square \eta=\square \chi, \\
& \square \square \chi=0 .
\end{aligned}
$$

From (4.6) it follows that $\square \chi$ is a harmonic field, i.e.,

$$
\square \chi=h \text {. }
$$

The most general solution to Eq. (4.7) is

$\chi=\frac{1}{4}\left[x^{-} \int^{x^{+}} d y^{+} f\left(y^{+}\right)+x^{+} \int^{x^{-}} d y^{-} g\left(y^{-}\right)\right]+\widetilde{h}$,

where $f$ and $g$ are defined via the general decomposition for a harmonic field in $1+1$ dimensions

$$
h(x)=f\left(x^{+}\right)+g\left(x^{-}\right),
$$

and where $\widetilde{h}$ is another harmonic field, i.e.,

$$
\square \widetilde{h}=0 \text {. }
$$

Using (4.8) and (4.5), we obtain for $A^{\mu}$ in (4.4)

$$
\begin{aligned}
& A^{0}(x)=\frac{1}{2}\left[x^{-} f\left(x^{+}\right)+\int^{x^{-}} d y^{-} g\left(y^{-}\right)\right]+\partial^{0} \hat{h}(x), \\
& A^{1}(x)=-\frac{1}{2}\left[x^{-} f\left(x^{+}\right)+\int^{x^{-}} d y^{-} g\left(y^{-}\right)\right]+\partial^{1} \hat{h}(x) .
\end{aligned}
$$

Here $\hat{h}(x)$ is still another harmonic field. Introducing (4.11) into (4.1b), one finds that the fields $f$ and $g$ are restricted to satisfy the equations

$$
\begin{aligned}
& \partial_{+} g\left(x^{+}\right)=0, \\
& \partial_{+} f\left(x^{+}\right)=-e \partial_{+} \phi(x) .
\end{aligned}
$$

Note that the right-hand side of $\mathrm{Eq}$. (4.13) does in fact only depend on $x^{+}$since, according to $(4.3 \mathrm{a}), \phi(x)$ is a harmonic field and, therefore, admits a decomposition of the form of (4.9).

From inspection of (4.11) it follows that translational invariance cannot be implemented by a unitary operator in the usual way, unless $f=g=0$. This leaves $A^{\mu}=\partial^{\mu} \hat{h}$ as the only possibility. However, this would imply $\pi_{1}=0$, which is inconsistent with the equal-time commutator $\left[A^{1}\left(x^{1}\right), \pi_{1}\left(y^{1}\right)\right]=i \delta\left(x^{1}-y^{1}\right)$, following from (2.7) for $a=0$. Hence, no operator solution compatible with the equal-time commutator algebra exists in this case.

\section{CONCLUDING REMARKS}

Using the Dirac-bracket formalism we have shown that the model defined in (1.3) may be systematically quantized within the operator approach.

We have studied the model for all values of the regularization parameter $a$ in the range $a \geq 0$. For $a>1$ the solution to the field equations could be expressed in terms of two decoupled free scalar fields.

For $a=1$ the model undergoes a drastic change which manifests itself in the appearance of new constraints, leading to a completely new commutator algebra. One of the new constraints expresses the vanishing of the field tensor. Furthermore, not only the divergence of the gauge current but the current itself vanishes, and thus the model remains consistent. From the point of view of the original theory defined by (1.1), the vanishing of the gauge current is a highly nontrivial effect produced by the interaction. For $a=1$ the $A^{\mu}$ propagator is finite and of course cannot be obtained via a limiting procedure from that of Ref. 1. An interesting consequence of the particular chiral coupling chosen in (1.1) is that for $a=1$ the $A^{\mu}$ field only propagates along the $x^{+}=$const branch of the light cone.

The $a=0$ case is what may be called the chiral Schwinger model. It turns out that no operator solutions compatible with the equal-time algebra exist. Nevertheless, a finite $A^{\mu}$ propagator can be constructed as the limit $a \rightarrow 0$ of the corresponding propagator for $a>1$. That this is not in contradiction with the singular behavior of the field operator $A^{\mu}$ for $a \rightarrow 0$ can be traced to the fact 
that in this limit the field $\sigma$ becomes a massless field quantized with the wrong sign, as seen from Eq. (2.18).

Note added in proof. After this paper was submitted for publication we learned that a similar analysis of the cases $a>1$ and $a=1$ had been carried out independently by Rajaraman. ${ }^{5}$ However, the presence of the constraint (3.3) was overlooked in Ref. 5, thus leading to the erroneous conclusion that there was some residual local gauge freedom left in the theory for $a=1$. This mistake was recognized by $\mathbf{R}$. Rajaraman (private communication). A brief comment on this point has also been included in a recent paper of the same author. ${ }^{6}$

\section{ACKNOWLEDGMENTS}

One of us (H.O.G.) is indebted to Institut für Theoretische Physik der Universität Heidelberg for the kind hospitality extended to him during the realization of this work. H.O.G. was also supported by Conselho Nacional de Desenvolvimento Cientifico e Tecnologico, Brazil.
*On leave of absence from Instituto de Fisica, Universidade Federal do Rio Grande do Sul, 90000 Porto Alegre, RS, Brazil.

${ }^{1}$ R. Jackiw and R. Rajaraman, Phys. Rev. Lett. 54, 1219 (1985).

${ }^{2}$ We use the same conventions and notations as in Ref. 1 . We take $\hbar=1$.
${ }^{3}$ P. A. M. Dirac, Lectures on Quantum Mechanics (Yeshiva University, New York, 1964).

${ }^{4}$ Wherever possible we omit time labels in the field arguments.

${ }^{5}$ R. Rajaraman, Phys. Lett. 154B, 305 (1985).

${ }^{6}$ R. Rajaraman, Report No. CERN-TH 4268, 1985 (unpublished). 